THE CERN ISR CONTROL SCHEME FOR ACCELERATION BY PHASE DISPLACEMENT

by

E. Ciapala, R. Keyser and S. Myers

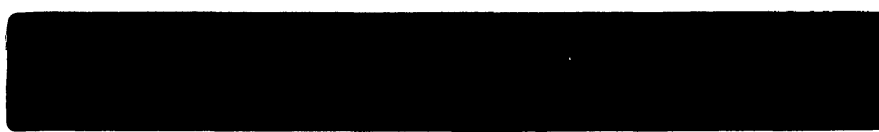

CM-P00064793

Presented at the 1979 Particle Accelerator Conference, San Francisco, 12-14 March 1979 


\section{THE CERN ISR CONTROL SCHEME FOR ACCELERATION BY PHASE DISPLACEMENT}

Edmond Ciapala, Richard Keyser and Stephen Myers*

Summary

In the CERN ISR high-intensity coasting beams are accelerated routinely from 26.6 to $31.4 \mathrm{GeV} / \mathrm{c}$ using the technique of phase displacement. The constraints imposed by this technique dictate a rather unusual control scheme. The total acceleration is performed in around two hundred steps; each incremental step in momentum is produced by sweeping empty RF buckets through the beam. The RF parameters are calculated and set by the control computer for each sweep. During each sweep the computer controls all magnetic elements synchronously so as to compensate for normal variations in the working line, the closed orbits and the beam position. This control is based on linear interpolation on the values held in a number of intermediate files between 26.6 and $31.4 \mathrm{GeV} / \mathrm{c}$. The differences in the power supply settings contained in these files produce the magnetic changes required to maintain the correct space charge tune shift and to compensate for magnetic saturation of the poles of the main bending magnets. The intermediate files are generated from the results of measurements made during the acceleration of a bunched beam using a different mode of the acceleration program.

\section{Introduction}

The control technique required for acceleration of a coasting beam by phase displacement is significantly different from that for synchronous acceleration of a bunched beam. In the latter case the bunched structure allows continuous monitoring of the beam position and the use of a feedback system to maintain the beam in the centre of the aperture. In addition, particularly in the later stages of acceleration, the beam size occupies only a small fraction of the available aperture.

For phase displacement the debunched nature of the beam greatly restricts beam monitoring possibilities and excludes the implementation of a continuous radial control feedback system. Also the stacked beam size completely fills the available aperture and remains large throughout the acceleration. Consequently small variations in the stack position or increases in the horizontal orbit distortion during acceleration result in intensity losses from the beam extremities. In addition, the continual pursuit of peak performance requires stack intensities which are close to the transverse coherent stability limit. Consequently small local reductions in the chromaticity (the $\Delta p / p$ of the stack is $23 \%$ ) during the course of acceleration may result in total beam loss. In view of the rather long time taken for stacking and acceleration (around four hours per ring) the control scheme must minimise the possibility of such losses.

\section{Control Requirements}

The control requirements for acceleration of beams by phase displacement are that the RF and magnet systems should be controlled in such a way as to accelerate the beam while maintaining the working line, the closed orbit and the beam extremities within preset limits. The control scheme may be broken down into two major parts. Firstly the pre-run preparations of computer files which contain the required increments to the magnet currents and secondly the calculations and hardware control needed to perform the actual acceleration of the beam.

“ CERN, Geneva, Switzerland.

\section{Pre-run preparation of computer files}

During acceleration the working line and the closed orbits are distorted due to saturation of the main magnet pole faces and due to the increase in the momentum of the beam. These two effects are fairly reproducible and may therefore be compensated by progressive excitation of the appropriate correction elements as a function of the beam momentum. The variations incurred by increasing the beam momentum are compensated by linearly increasing (with momentum) the magnet currents of all lattice elements such as quadrupoles, sextupoles, low beta quadrupoles etc. However the optical aberration due to magnetic saturation and other non-linearities must be measured and compensated as a function of beam momentum. To this end, low intensity $(<0.1 \mathrm{~A})$ bunched beams are accelerated synchronously in steps of around $1 \mathrm{GeV} / \mathrm{c}$ through the required momentum range. With such low intensities the incoherent tune shift is completely negligible. After each step the working line and the closed orbits across the aperture are measured and corrected. The magnet current increments required for these corrections are stored in computer files along with the measured beam momentum. Subsequently, linear interpolation with momentum between these files gives the magnet current increments needed to keep the working line and the closed orbits constant during acceleration. The use of the higher harmonic cavity ${ }^{l}$ stabilises the bunches against coupled bunch oscillations and allows the tight bunch structure to be maintained for several hours. The same bunched beam may therefore be used for all measurements between 26.6 and $31.4 \mathrm{GeV} / \mathrm{c}$. The reproducibility of the integrated magnetic fields, improved by systematic cycling ${ }^{2}$ of all relevant magnets allows that the measured corrections need only be checked at most a few times per year.

The working line is also strongly distorted by the presence of the high-intensity beam. Since the magnitude of this 'incoherent tune shift' depends on the density distribution of the beam, it is not reproducible from run to run. Nevertheless for a beam whose density distribution remains constant during acceleration the magnet current increments (usually pole-face windings) necessary to compensate for the incoherent tune shift are independent of beam momentum. This results from the fact that the incoherent tune shift is inversely proportional to momentum whereas the magnet current increments required to give a fixed tune shift are directly proportional to momentum. Consequently, in practice a first approximation to continuous space charge compensation is achieved by simply maintaining the magnet current increments derived for space charge compensation of the beam at $26.6 \mathrm{GeV} / \mathrm{c}$.

The variations in the density distribution of the beam (displacements of the centre of gravity, the form of the distribution and the total beam intensity) during acceleration occur quite slowly and may therefore be measured and compensated before excessive beam losses can occur. During the actual phase displacement sweep the density distribution is obtained by measuring (as a function of the revolution frequency) the amplitude of the pick-up signal produced by the passage of the empty buckets through the beam. The measured points are stored $^{3}$ and fed to the computer at the end of the scan. This data is used to correct the changes in the incoherent tune shift due to the variations in the density distribution of the beam. Alternatively the density profile may also be obtained from the longitudinal Schottky scan ${ }^{4}$. The use of this device is limited to coasting beams without longitudinal modulation by the 
empty buckets.

The working line is also transiently distorted to a small extent during the traversal of the beam by the RF bucket. At present this effect cannot be measured and is not controlled.

\section{Control of the actual acceleration}

For each ISR operational working line a data base exists in which are stored all relevant lattice parameters. The control computer also has access to a high precision measurement of the main bending field 5 and hence the central momentum $\left(p_{C}\right)$.

The frequency spread of the stack is simply

$$
\Delta f_{R F}=\frac{h|\eta| f_{r e v} \Delta p_{s t}}{p_{C}}
$$

where $h$ is the $R F$ harmonic number and $\Delta p_{s t}$ is the full momentum width of the stack. Additional momentum 'blow-up' and beam losses occur if the RF voltage is switched on or off close to the edges of the beam. Consequently the RF must sweep a range greater than the beam width. Measurements and computations have shown that the effects of switching transients are negligible when the sweep starts or ends at greater than around four full bucket widths from the edge of the beam. In the ISR this condition can always be satisfied by sweeping the momentum width of the full aperture $\left(\Delta p_{a p}\right)$. The frequency at which the $R F$ is switched on is then simply

$$
f_{\text {start }}=h f_{\text {rev }}\left\{i+\frac{\eta}{2 p_{c}} \Delta p_{a p}\right\}
$$

The required rate of frequency change is

$$
\frac{d f_{R F}}{d t}=h\left\{\frac{c}{2 \pi R_{C}}\right\}^{2} \frac{\eta}{\gamma E_{O}} v_{R F} \sin \phi_{S}=c\left(p_{C}\right) v_{R F} \Gamma
$$

where $\Gamma=\sin \phi \mathrm{s}^{\circ}$

Before each accelerating sweep the values of $C\left(p_{C}\right), f_{\text {start }}$ and $\Delta f_{R F}$ are calculated and transmitted to the $R F$ hardware along with $V_{R F}$ and $\Gamma$ which are given as input to the control program. The beam momentum increment which will result from each sweep is

$$
\overline{\Delta p}_{\text {in }}=\frac{16}{(2 \pi)^{3 / 2}} \frac{\mathrm{p}_{\mathrm{C}}}{\beta} \alpha(\Gamma) \sqrt{\frac{\mathrm{v}_{\mathrm{RF}}}{\gamma \mathrm{hn \textrm {E } _ { \mathrm { O } }}}}
$$

where $\alpha(\Gamma)$ is the area ratio of a moving to a stationary bucket and is a complex function for which no simple equation exists. For this reason the tabulated values of $\alpha(\Gamma)$ have been fitted as a polynomial in $\Gamma$.

In equations (3) and (4) $\mathrm{E}_{0}$ is the rest energy of the particle. Deuterons ${ }^{6}$ as well as protons have been accelerated using the same technique.

Before each accelerating sweep the expected momentum increment is calculated from (4) and this value, added to the actual momentum is used for the interpor lation between the set of intermediate files described in the previous section. This gives the current increments which should be made to each magnet (during the $\mathrm{RF}$ sweep) so as to compensate for the shift in the beam position, the deformation of the working line and the distortion of the closed orbit. In total around 70 power supplies are controlled during a sweep.

\section{RF Hardware for Phase Displacement}

The drive to the RF cavities is generated as shown in Fig. 1. A voltage-controlled crystal oscillator (VCXO) operates at $20 \mathrm{MHz}$ and has a linear frequency range of approximately $7 \mathrm{kHz}$. The output of the VCXO is mixed with a $29.5 \mathrm{MHz}$ signal from a synthesizer to produce the $9.5 \mathrm{MHz}$ required for the cavities. The VCXO is controlled by separate program generators for stacking and phase displacement.

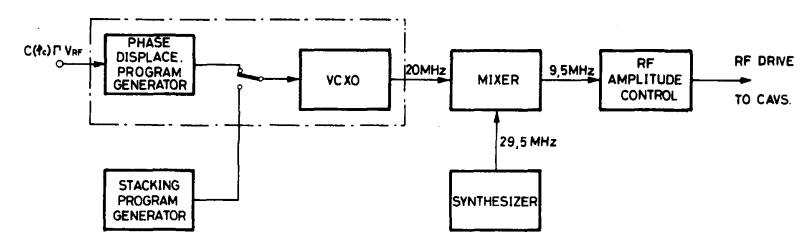

Fig. 1. Simplified diagram of drive to RF cavities

The efficiency of phase displacement is greatly reduced by the presence of phase noise on the $R F$ at or near the synchrotron frequency ${ }^{7}$. In the ISR with the standard RF parameters used for acceleration the synchrotron frequency is close to the mains frequency which is $50 \mathrm{~Hz}$. The effect of the noise is considerably less during stacking because phaselock is used. The generation of the frequency program for phase displacement is simpler than that for stacking and fewer external analog and timing signals are required. A completely separate program generator mounted close to the VCXO can therefore give better noise immunity for phase displacement. The program generator is shown in Fig. 2 .

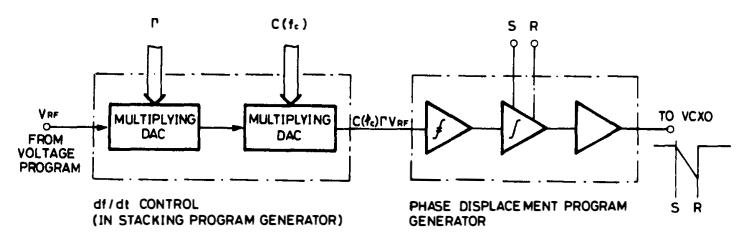

Fig. 2. Simplified block diagram of program , generator for phase displacement

The voltage analog of the product $c\left(p_{C}\right) \Gamma V_{R F}$ is produced in the normal stacking frequency program generator by two multiplying digital to analog converters. This voltage is filtered and integrated to give the required linear sweep. The program generator and the VCXO are mounted in close proximity inside a shielded box and are fed by external low-ripple power supplies.

The phase noise may be measured by using a phase detector to compare the RF signal with a pure reference source at the same frequency. Figure 3 shows the lowfrequency phase noise on the RF recorded on a lowfrequency spectrum analyser for each program generator.

The phase-displacement program gives a reduction of around $15 \mathrm{~dB}$ at $50 \mathrm{~Hz}$ and its harmonics. The corresponding equivalent peak phase modulation at $50 \mathrm{~Hz}$ is less than 1:. For a $30 \mathrm{~A}$ stack this corresponds to a reduction in the loss per phase-displacement sweep from around $30 \mathrm{~mA}$ to $3 \mathrm{~mA}$. 


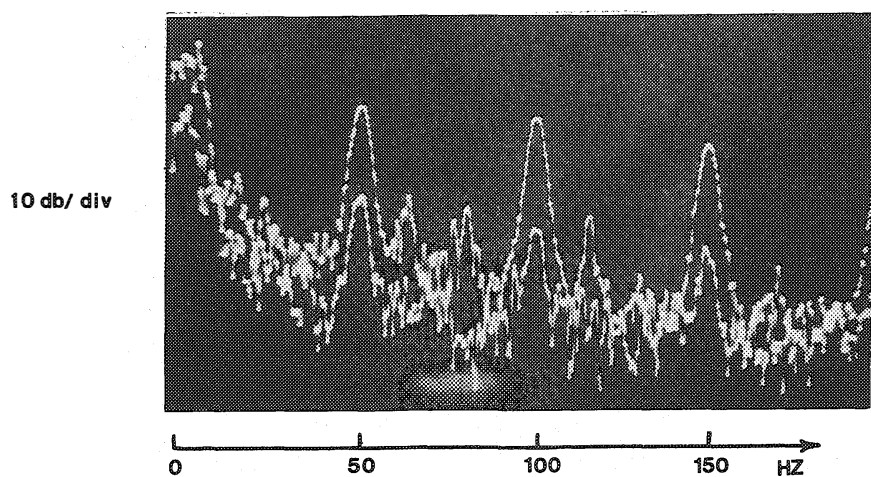

Fig. 3. Spectra of low-frequency phase noise. upper trace : stacking program lower trace : phase-displacement program.

\section{Single pulse acceleration}

Single pulse bunched beams are accelerated in order to measure machine parameters such as the working line and the closed orbit at intermediate energies (Section 2). A separate radial control system is used. The block diagram is shown in Fig. 4.

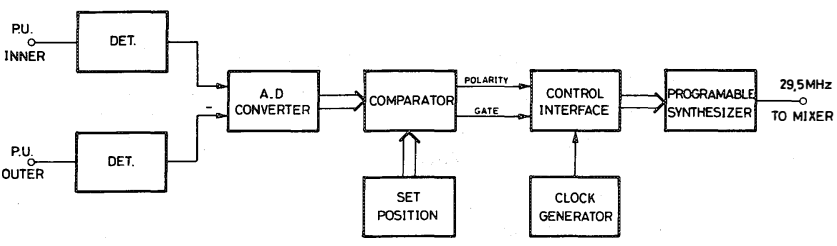

Fig. 4. Radial control for acceleration of single pulses.

The normal synthesizer operating at $29.5 \mathrm{MHz}$ is replaced by a programmable synthesizer which has phase continuity over the frequency range required. The radial position is obtained from horizontal pick-up signals. Any deviation from the required set position is detected by the comparator. The interface gates clock pulses to the synthesizer to step the frequency in such a way that the beam returns to the set position. A 'dead' zone is allowed in the comparator to provide stability. The beam may be moved radially in the vacuum chamber at any intermediate energy to permit working-line or closed-orbit measurements by varying the set position.

\section{Computer Programs}

The increments to the magnet power supplies at various energies are kept in standard power supply setting files. A preliminary program accepts a list of power supply file names and creates a master file containing the names in this list together with information extracted from the files in preparation for the acceleration program proper.

The acceleration program, $\mathrm{ACCZ}$, runs on the ARGUS computer and requests the NORD-10 computer, which is dedicated to power supply control, to read the current power supply values or to increment them by given amounts. The incremental process is such that the rate of change of current in each magnet is proportional to the total required change in that magnet. The setting and triggering of the RF system is performed by ACCZ through CAMAC modules attached to the ARGUS via a parallel bus. From these modules data are transmitted serially to the RF system in the auxiliary buildings. This system was installed early in the life of the ISR before the serial CAMAC highway had been adopted.

The computer program sets the $R F$ into the correct mode, calculates and sets up $C\left(p_{C}\right), V_{R F}, \Gamma, f_{\text {start }}$ and $\Delta f_{R F}$, as described earlier, and then triggers the sweep. The reset of the $R F$ is generated automatically by the RF program generator at the end of the sweep and this also informs the ARGUS by means of an interrupt.

The program pauses awaiting operator action whenever the beam loss during a sweep exceeds a settable threshold or whenever the momentum of the beam passes a value corresponding to one of the data files. In these cases the program first checks that the power supplies are actually at their expected values and signals disscrepancies. This check is also carried out when the program terminates. It is not carried out on every sweep since this would considerably increase the total acceleration time. The program can also be interrupted by the operator at the end of a sweep by a switch. Whenever the program is interrupted the operator may change certain program parameters, e.g. for top-of-stack control (see below) or RF parameters.

Any difference between the actual RF bucket. area ${ }^{2}, 7$ and that calculated by the program causes the stack to drift in the vacuum chamber. This is automatically compensated by calculating the top-of-stack position on each $R F$ sweep and keeping it between chosen radial limits. If the top-of-stack exceeds the upper limit the power supplies are incremented without sweeping the RF. If the top-of-stack drops below the lower limit, the RF is swept alone.

The program maintains a log during the acceleration recording on a disc file the momentum, beam current and top-of-stack position after every sweep.

As mentioned earlier the program has a special mode whereby the power supply settings are incremented by the difference between two working files. The program does not control the RF which is autonomously tracking the bunched beam to maintain its position in the chamber.

The program ACCZ has an initialisation phase lasting some $20 \mathrm{~s}$. Each sweep takes 6 to $10 \mathrm{~s}$.

\section{References}

1. H. Frischholz et al., Longitudinal Stabilization of Bunched Beams in the ISR by a higher harmonic cavity, Proc. Internat. Conf. on High Energy Accelerators, Serpukhov, July 1977.

2. C. Fischer et al., Performance of the CERN ISR at $31.4 \mathrm{GeV}$, To be presented at this conference. 3. D. Kemp, E. Peschardt and A. Vaughan, on line $Q$ measurement during phase displacement acceleration in the CERN ISR, To be presented at this conference. 4. J. Borer et al., Non-destructive Diagnostics of Coasting Beams with Schottky Noise, Proc. Internat. Conf. on High Energy Accelerators, Stanford, 1974. 5. K.N. Henrichsen and J.-P. Gourber, The Computer Controlled System for the Forecast of Beam Parameters in the CERN ISR, Proc. Internat. Conf. on High Energy Accelerators, Geneva, 1971.

6. S. Myers, Deuterons in the ISR, CERN-ISR-OP/77-28, April 1977 .

7. S. Myers and E. Peschardt, The Efficiency of Acceleration by Phase Displacement in the Presence of RF Noise, To be presented at this conference. 